TIPA. Travaux interdisciplinaires sur la parole et le langage

$28 \mid 2012$

Linguistique expérimentale

\title{
Dimension esthétique des voix normales et dysphoniques: Approches perceptive et acoustique
}

Melissa Barkat-Defradas, Coralie Busseuil, Oriane Chauvy, Fabrice Hirsch, Camille Fauth, Joana Révis et Benoît Amy de la Bretèque

\section{OpenEdition}

Édition électronique

URL : http://journals.openedition.org/tipa/204

DOI : $10.4000 /$ tipa.204

ISSN : 2264-7082

Éditeur

Laboratoire Parole et Langage

Référence électronique

Melissa Barkat-Defradas, Coralie Busseuil, Oriane Chauvy, Fabrice Hirsch, Camille Fauth, Joana Révis et Benoît Amy de la Bretèque, " Dimension esthétique des voix normales et dysphoniques : Approches perceptive et acoustique », TIPA. Travaux interdisciplinaires sur la parole et le langage [En ligne], 28 | 2012 mis en ligne le 29 octobre 2012, consulté le 10 décembre 2020. URL : http://journals.openedition.org/ tipa/204 ; DOI : https://doi.org/10.4000/tipa.204

Ce document a été généré automatiquement le 10 décembre 2020.

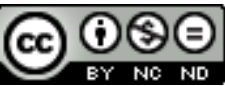

La revue TIPA. Travaux interdisciplinaires sur la parole et le langage est mise à disposition selon les termes de la licence Creative Commons Attribution - Pas d'Utilisation Commerciale - Pas de Modification 4.0 International. 


\title{
Dimension esthétique des voix normales et dysphoniques: Approches perceptive et acoustique
}

\author{
Melissa Barkat-Defradas, Coralie Busseuil, Oriane Chauvy, Fabrice \\ Hirsch, Camille Fauth, Joana Révis et Benoît Amy de la Bretèque
}

\section{Introduction}

1 La question de l'esthétique de la voix parlée suscite un intérêt grandissant dans la communauté des chercheurs en parole et des professionnels de la voix - phoniatres et orthophonistes - impliqués dans les processus de rééducation vocale (Bruckert et al., 2010, Liu \& Wu, 2011). Ceci s'explique, entre autres, par le fait que la voix est un outil de communication et d'interactions sociales qui influe fortement sur l'image qu'un individu peut avoir de son interlocuteur. Cet effet relatif au stéréotype de l'attractivité vocale renvoie à l'idée que " what sounds beautiful is good " ${ }^{1}$ (Zuckermann \& Driver, 1989) et laisse à penser que la pathologie vocale est perçue comme négative. Toutefois, nous pensons que certaines voix pathologiques peuvent être perçues comme séduisantes. En effet, bien que le jugement que l'on porte sur les voix tienne à des paramètres tout à fait personnels, certaines d'entre elles, quoique franchement pathologiques, s'avèrent appréciées d'un large public, que l'on pense par exemple aux voix de l'acteur Richard Bohringer, à celle de la comédienne Jeanne Moreau, ou encore à celle du chanteur Garou. Pourtant, à l'exception d'une étude menée sur la voix chantée (Seidner \& Büttner, 1999), la dimension esthétique des voix pathologiques - dans la modalité parlée - est une problématique qui, à notre connaissance, n'a encore jamais été abordée dans la littérature. L'objectif du présent travail est précisément d'évaluer, par le biais d'une expérience perceptive, le caractère esthétique des voix dysphoniques et de déterminer les indices acoustiques ayant un effet sur le jugement positif des auditeurs. 


\section{Voix et image sociale}

2 Le jugement esthétique des voix est fortement conditionné par le milieu culturel en fonction des valeurs véhiculées par la société à une période donnée et des représentations mentales que les locuteurs ont de ces voix (Barkat-Defradas \& Dufour, 2007). Les critères d'appréciation vocale obéissent ainsi à des codes différents selon les sociétés et les cultures (Cornut, 2005). À titre d'exemple, citons le registre de la voix aiguë qui, associé à la féminité dans les pays occidentaux, est aujourd'hui socialement inacceptable pour les hommes européens alors que l'utilisation de ce même registre vocal est parfaitement normal en Afrique du Nord ou en Orient (Le Huche \& Allali, 1990). Dans le même ordre d'idée, les femmes japonaises en position d'autorité troquent leur voix naturelle pour une « voix sociale » calquée sur le modèle masculin (Smith, 1992). Outre les facteurs culturels et géographiques, les critères esthétiques de la voix évoluent également avec le temps (Barberis \& Barkat-Defradas, 2011 ; Boula de Mareuil et al., 2008; Gillie, 2009 ; Pemberton et al., 1998 ; Yuasa, 2010). Le jugement vocal répond donc à des critères subjectifs de goût personnel, culturel, de genre, d'époque et on ne peut définir la qualité d'une voix de façon absolue. Néanmoins, bien qu'il ne puisse y avoir de consensus sur ce que pourrait être une "belle voix", des travaux récents montrent que la qualité vocale a une profonde influence sur les auditeurs (Bruckert et al., 2010). C'est ainsi qu'un certain nombre d'études se sont intéressées aux stratégies vocales mises en œuvre lors des rapports de séduction et à leurs effets sur l'Autre. Berry (1990) et Ciceri (2001) ont montré qu'en Europe, l'utilisation d'une voix infantile par les femmes (i.e. babyish voice) facilite la prise de contact lors de la recherche d'un partenaire masculin. En outre, la voix, en tant que caractère sexuel secondaire, véhicule des informations essentielles tant aux niveaux biologique, psychologique que social (Karpf, 2008). La plupart des études qui se sont intéressées à l'évaluation perceptive de la voix ont montré qu'il était possible - à partir des seules caractéristiques vocales - d'inférer un certain nombre de caractéristiques physiologiques (Krauss et al., 2002) telles que : le sexe (Lieberman, 1984 ; Mullenix et al., 1995 ; l'âge (Ryan \& Burk, 1974 ; Horii, \& Ryan, 1981 ; Neiman \& Applegate, 1990 ; Braun, 1996 ; Loredana et al., 2000) ou la taille et le poids (Lass \& Davis, 1976 ; Lass et al., 1980 ; Künzel, 1989 ; van Domelen \& Moxness, 1995 ; Evans et al., 2006). D'autres travaux, conduits pour les plus récents dans le champ de la biologie évolutive, ont également établi une corrélation entre d'une part, la qualité vocale perçue et d'autre part, l'attractivité faciale (Collins, 2000 ; Collins, 2003 ; Feinberg et al., 2005 et 2008 ; Zuta, 2007 et 2009) ; l'orientation et/ou le comportement sexuel (Smith et al., 2003 ; Pierrehumbert et al., 2004) ; Hughes et al., 2004 ; Puts et al., 2006 et 2008 ; Apicella et al., 2007 ; Vukovic, et al. 2011) ; certains traits de la personnalité (Hart, 1971 ; Brown et al., 1973 ; Smith et al., 1975 ; Apple et al., 1979 ; Benjamin, 1981 ; Streer, 1974 ; Ray, 1986 ; Gregory et al., 1993 ; Gregory, 1994 ; Smith \& Schaffer, 1995 ; Hughes \& Gallup, 2008 ; Puts et al., 2008 ; Barkat-Defradas \& Raymond, 2011) ou encore certaines caractéristiques relatives au statut socio-économique des locuteurs (Harms, 1963 ; Ellis, 1967).

Outre la transmission de messages verbaux à contenu informationnel, la voix - outil privilégié de la communication humaine - véhicule également une quantité non négligeable d'informations extralinguistiques, certaines d'entre elles, relatives par exemple à l'état de santé mentale du locuteur, pouvant avoir des conséquences importantes sur la vie sociale des personnes concernées (House \& Andrews, 1987 ; Scott et al., 1997 ; White, et al., 1997). 
Une dysphonie - et a fortiori une aphonie - altère la communication. En effet, les désordres vocaux, qu'ils soient engendrés par une pathologie, ou secondaires à une intervention chirurgicale, ont un impact fort sur l'état psychologique et sur la vie sociale des personnes concernées. Des études récentes se sont intéressées aux représentations associées aux voix pathologiques et notamment à l'image sociale qu'elles véhiculent (Revis et al., 2009, Louvet, 2010; Raymond, 2010). Ainsi, alors que les voix « lisses et sans aspérités » sont associées à des locuteurs jeunes, fertiles et en bonne santé (Bruckert et al., 2008), les locuteurs dysphoniques sont décrits comme plus âgés, plus fatigués, plus déprimés et moins compétents que les sujets sains (en termes de niveau d'éducation et de compétence professionnelle), et ce, dès les stades légers de la pathologie (Privat, 2009). De plus, il a été montré que plus la dysphonie est sévère, plus l'évaluation est négative (Altenberg \& Ferrand, 2006). Enfin, pour en ce qui concerne le jugement hédonique, les voix saines sont toujours évaluées comme plus agréables que les voix dysphoniques, notamment sévères (Raymond, 2010).

\section{Évaluation de la qualité vocale}

5 La qualité vocale est une notion très complexe communément définie par défaut comme ce qui différencie deux productions vocales ayant le même contenu lexical (Payri, 2000). Pour certains auteurs, la qualité vocale comprend toutes les caractéristiques de la voix : « le timbre, la hauteur, l'intonation, l'articulation, l'intensité, le rythme et la nasalité » (Garnier et al., 2005 :152). Pour d'autres, cette notion renvoie au timbre vocal perçu, à la « couleur de la voix» (Konopczynski, 2005 et 2007). De façon générale, l'appréciation de la qualité vocale dépend principalement de deux critères : (i) le domaine d'expression vocale (i.e. caractère normal vs pathologique de la voix, voix parlée vs voix chantée...) et (ii) le contexte communicationnel (i.e. parole spontanée vs parole de laboratoire). Dans la pratique clinique, l'évaluation de la qualité vocale vise la catégorisation des voix en fonction de différents paramètres. Elle est fondée d'une part sur une évaluation perceptive (i.e. à l'oreille) de la qualité vocale et, d'autre part, sur une analyse instrumentale basée sur des mesures acoustiques et aérodynamiques des sons de parole.

\section{Évaluation perceptive}

6 L'évaluation perceptive repose sur le jugement humain et sur les capacités de l'auditeur à évaluer la qualité d'une voix. Sa fiabilité est souvent controversée. Une méta-analyse conduite sur 57 articles publiés entre 1951 et 1990 a montré un niveau de reproductibilité allant de 18 à $100 \%$, avec des protocoles et méthodes d'analyses statistiques si différents que la comparaison des résultats est quasi impossible (Kreiman et al., 1993). Une méthodologie stricte et reproductible doit être mise en place pour améliorer la fiabilité de l'évaluation perceptive et pallier son caractère subjectif. Elle passe par le choix de variables qualitatives, puis par la mise en place d'une échelle quantitative. En clinique, la nature d'une voix est décrite en termes d'impressions auditives: hauteur, éraillement, raucité, souffle, craquements, etc. Le choix de ces termes a été largement discuté et débattu dans la littérature (De Bodt et al., 1996 ; Hammarberg et al., 1980).

7 Dans la mise en place d'un protocole d'évaluation perceptive, le choix du jury est également fondamental. Sa qualité est évaluée en termes de fiabilité, laquelle correspond à la reproductibilité du jugement entre les auditeurs (i.e. agrément interauditeur) et par 
l'auditeur lui-même, lors de plusieurs sessions d'écoute (i.e. agrément intra-auditeur). Afin d'améliorer la fiabilité de l'évaluation, plusieurs sessions d'écoute sont organisées pendant lesquelles les voix sont présentées dans un ordre aléatoire (Kreiman, 1996). Il convient en effet de contrôler le contexte dans la mesure où ce dernier est un facteur d'influence d'importance : une voix moyennement dysphonique parait plus altérée si elle est présentée après une voix normale qu'après une dysphonie sévère (Goldstone, 1998). Menée dans des conditions expérimentales dûment contrôlées, l'analyse perceptive facile à mettre en œuvre, accessible à tout clinicien et peu coûteuse - est la seule capable de rendre compte des caractéristiques perceptives de la voix. En revanche, elle est chargée de plusieurs biais intrinsèques qui la rendent imparfaite voire insuffisante. En effet, de nombreux facteurs influençant le jugement perceptif ne peuvent être tout à fait contrôlés parmi lesquels : l'état émotionnel de l'auditeur au moment de l'évaluation, ses valeurs esthétiques, sa langue maternelle et/ou son dialecte d'origine, la manière dont il conçoit l'échelle de mesure, etc. (Révis et al., 1999). Concrètement, une analyse perceptive de la qualité de la voix est menée par un panel de spécialistes (thérapeutes de la voix, phoniatres et orthophonistes), lesquels effectuent une description analytique des caractéristiques vocales sur la base d'échelles standardisées telles que: le CAPE-V (Kempster et al., 2009) ou, le plus souvent, l'échelle GRBAS (Hirano, 1981). La GRBAS est une échelle perceptive basée sur l'évaluation de cinq paramètres acoustiques (Hirano, 1981 ; 1989) : (i) le grade global de la dysphonie (Grade), (ii) le degré de raucité de la voix ( Roughness), (iii) le souffle (Breathiness), (iv) la faiblesse vocale ou asthénie vocale (Aesthenia ) et (v) le forçage vocal (Strain). Chacun de ces paramètres est côté selon quatre degrés de sévérité : 0 (normal), 1 (légèrement altéré), 2 (altéré) et 3 (sévèrement altéré). Cette échelle peut être appliquée lors de la production d'une voyelle tenue, d'une phrase ou d'un texte généralement lus.

\section{Analyse instrumentale}

L'analyse instrumentale est conçue pour qualifier et surtout quantifier les dysphonies à partir de mesures acoustiques et/ou aérodynamiques (Baken et al., 2000). Ces mesures sont le plus fréquemment réalisées sur une voyelle tenue - en général le /a/ - à l'aide de différents capteurs conçus pour enregistrer et étudier de multiples paramètres de la production de parole (Ghio et al., 2008). Il est toutefois souvent nécessaire de combiner différentes mesures complémentaires afin de tenir compte de la dimension multidimensionnelle de la production vocale (Ghio et al., 2007). Les mesures acoustiques (i.e. fréquence et amplitude; jitter et shimmer; analyse spectrale) révèlent les caractéristiques audibles de la dysphonie. Il s'agit principalement des mesures de la fréquence fondamentale et de l'intensité, de leur stabilité, ainsi que de l'analyse du spectre du son émis. Les mesures aérodynamiques, sans être à proprement parler des mesures de la voix, permettent d'évaluer les caractéristiques biomécaniques du système pneumo-phonatoire. Il s'agit principalement de mesures de débit, de pression et d'efficacité glottique.

9 Dans le cadre de l'évaluation des dysphonies, l'approche instrumentale multiparamétrique, rendue possible par l'existence d'outils d'expertise fiables (Giovanni et al., 1992), offre une approche complémentaire à l'approche perceptive qui est, par nature, considérée comme 'l'étalon-or', dans la mesure où la voix est avant tout un phénomène perceptif tant du point de vue de celui qui l'émet (le sujet parlant) que de 
celui qui l'évalue (le clinicien, mais également l'entourage). C'est pourquoi les résultats présentés dans le cadre de cette étude procèdent (i) d'une évaluation perceptive de la qualité vocale conduite, dans un premier temps, par des experts (catégorisation des voix selon l'échelle de Hirano) et, dans un second temps, par des sujets naifs (évaluation du caractère plus ou moins agréable des voix) et, (ii) de l'analyse acoustique des voix jugées comme les plus « belles » par les auditeurs non experts.

\section{Étiologie des dysphonies}

Le Huche (2001 : 47) définit la dysphonie comme :

un trouble momentané ou durable de la fonction vocale ressenti comme tel par le sujet lui-même ou par son entourage. Elle se traduit le plus souvent, mais non obligatoirement, par une altération d'un ou plusieurs des paramètres acoustiques de la voix, soit, par ordre de fréquence, du timbre, de l'intensité et de la hauteur tonale.

11 Au plan clinique, on parle de dysfonction vocale qu'il y ait une lésion organique ou non (mauvaise utilisation des organes de la phonation, c'est-à-dire malmenage ou surmenage). Les dysphonies ont des causes liées non seulement aux cordes vocales (par exemple du fait d'une inflammation), mais aussi à l'appareil vocal dans son ensemble (soufflerie et résonateurs). Le corps (par le biais de la posture, du tonus musculaire), et bien sûr le psychisme peuvent également être à l'origine d'un trouble vocal. Les origines des dysphonies s'avèrent ainsi variées. Teston (2001) propose de distinguer les dysphonies d'origine neurologique (provoquées par l'état neuromoteur du patient) des dysphonies d'origine morphologique (résultant de changements anatomiques de la glotte). Dans ce dernier cas, il peut s'agir soit de lésions bénignes des cordes vocales (nodules, polypes, kystes) généralement provoquées par un forçage vocal; soit d'inflammations de l'ensemble des cordes vocales qui peuvent s'installer de manière permanente; soit encore de traumatismes post-chirurgicaux. Dans tous les cas, l'impression auditive qui se dégage est que ces voix pathologiques sont fréquemment perçues comme plus graves, plus éraillées, plus rauques que les voix saines (Teston, document électronique). Or, une étude conduite dans le domaine de la qualité vocale dans la musique pop rock révèle que si la raucité est bien perçue comme le symptôme d'une maladie, elle est également une forme d'expression appréciée des auditeurs (Siedner \& Büttner, 1999). Dans ce travail, les auteurs ont fait écouter à des juges des échantillons vocaux de différents chanteurs appartenant à divers genres musicaux. Chaque participant devait évaluer chacun des échantillons sonores en choisissant entre : voix "en bon état " ou voix «malade»; "voix « rauque » ou voix « pas rauque » avant de dire s'ils aimaient ou non la voix qui leur était présentée. Les résultats montrent que pour l'ensemble des participants, raucité et maladie sont une seule et même chose ; c'est-à-dire que les voix rauques sont considérées par une écrasante majorité comme pathologiques, alors que les voix qui ne le sont pas sont perçues comme saines. Mais, pour ce qui concerne l'évaluation du caractère esthétique des échantillons sonores, l'ensemble des juges a déclaré avoir aimé les voix qui étaient, sans équivoque, légèrement rauques. Ce résultat nous a conduits à nous interroger sur la dimension esthétique accordée au paramètre de la raucité (i.e. Roughness) dans le contexte de la voix parlée normale et pathologique. 


\section{Hypothèses de recherche}

12 Le premier objectif de ce travail ${ }^{2}$ est de vérifier si certaines voix dysphoniques de locuteurs masculins peuvent être qualifiées de séduisantes par un éventail de juges de sexe féminin. Ce choix est motivé par le fait que les travaux en éthologie animale nous apprennent que chez la plupart des espèces, le rôle de la séduction est dévolu au mâle (Allainé et al., 2010). Parallèlement, il a été démontré que les femmes sont sensibles à la voix masculine, comme critère d'attirance sexuelle dans leur choix du partenaire (Evans, 2008). L'objectif de cette étude est de se pencher sur la question du potentiel de séduction des voix masculines dans le contexte de la pathologie vocale. C'est pourquoi, le groupe de juges est exclusivement composé de sujets de sexe féminin, les échantillons vocaux à évaluer étant produits uniquement par des locuteurs de sexe masculin. Le second objectif de cette recherche visera à mettre en évidence certaines des caractéristiques acoustiques des voix pathologiques les plus séduisantes, afin d'observer ce qui les distingue des voix dysphoniques jugées comme moins attrayantes. On sait que, lorsqu'elles doivent choisir parmi plusieurs voix masculines non pathologiques celles qu'elles perçoivent comme les plus séduisantes, les femmes ont une nette préférence pour les voix les plus graves (Collins, 2000 ; Bruckert et al., 2006). On sait également qu'un certain nombre de voix dysphoniques sont elles-mêmes plus graves que la moyenne (Teston, document électronique). Par conséquent, notre principale hypothèse est que certaines voix présentant une dysphonie légère et dont le timbre est plus grave que les voix saines, pourraient être perçues comme plus séduisantes que des voix non pathologiques. La seconde hypothèse est que certaines caractéristiques vocales, comme la raucité, pourraient conférer aux voix dysphoniques de type R1 une certaine dimension esthétique.

\section{Méthode expérimentale}

\subsection{Acquisition des stimuli vocaux}

13 Pour vérifier nos hypothèses, des échantillons sonores produits par des locuteurs de sexe masculin dysphoniques $(\mathrm{n}=31)$ et non dysphoniques $(\mathrm{G} 0, \mathrm{n}=31)$ ont été sélectionnés et/ ou enregistrés (voir Tableau 1). Notons que les échantillons de voix pathologiques (G1 et G2) ont été recueillis dans la banque de données EVA du CHU La Timone de Marseille. Pour chaque voix pathologique, nous disposions d'un enregistrement du premier paragraphe du texte de la «La Chèvre de Monsieur Seguin» (parole lue) et d'enregistrements de [a] tenus. Les voix saines ont été enregistrées par nos soins à l'aide d'un équipement semi-professionnel (Zoom H2, 22Khz, 16bits, mono). Les sujets, en position debout (micro sur pied placé à $15 \mathrm{~cm}$ de la bouche) avaient pour consigne de lire le plus naturellement possible et à intensité normale le même extrait littéraire que précédemment.

14 Avant le démarrage de l'enregistrement, les sujets pouvaient se familiariser aussi longtemps qu'ils le souhaitaient avec le texte. Chaque sujet était enregistré trois fois, nous avons sélectionné la meilleure version (i.e. absence de bruit de fond parasite, d'hésitations, etc.). Avant d'être soumises au jugement du jury naïf, les voix saines et dysphoniques ont fait l'objet d'une évaluation subjective par un jury d'experts composé

TIPA. Travaux interdisciplinaires sur la parole et le langage, 28 | 2012 
d'orthophonistes et d'un médecin phoniatre. Ce jury a déterminé par consensus et pour l'ensemble des voix les composantes $G$, $R$ et $B$ de l'échelle GRBAS de Hirano. Les composantes relatives à l'asthénie (A) et au forçage vocal (S) n'ont pas été étudiées ici, leur fiabilité en termes d'agrément interauditeur ayant été remise en cause dans la littérature (Revis, 2004).

Tab. 1. Répartition des voix par grade et par âge * critère d'inclusion /âge : 20-60 ans (afin d'éviter les effets de mue ou de presbyphonie)

\begin{tabular}{|l|l|l|}
\hline Grade & Effectifs/62 & âge moyen* \\
\hline G0 & $n=31$ & 39,3 \\
\hline G1 & $n=14$ & 43,7 \\
\hline G2 & $n=17$ & 40,2 \\
\hline
\end{tabular}

Le critère plus particulièrement étudié dans le cadre de cette étude étant relatif à la raucité vocale et à l'éventuel attrait qu'elle suscite, le Tableau 2 suivant fournit, pour chaque grade, la proportion de voix évaluées R0, R1, R2 par le jury d'experts.

Tab. 2. Proportion (en \%) de voix évaluées comme rauques en fonction du grade

\begin{tabular}{|l|l|l|l|}
\hline Grade & R0 & R1 & R2 \\
\hline G0 & $0 \%$ & $0 \%$ & $0 \%$ \\
\hline G1 & $42,86 \%$ & $50 \%$ & $7,14 \%$ \\
\hline G2 & $17,65 \%$ & $17,65 \%$ & $64,7 \%$ \\
\hline
\end{tabular}

Les pathologies représentées dans cette étude sont répertoriées dans le Tableau 3 ("Causes et effectifs des pathologies vocales»). Seuls ont été retenus les cas de dysphonies fonctionnelles ou organiques bénignes. Les lésions ORL malignes n'ont pas été retenues dans la mesure où il s'agit d'atteintes trop lourdes pour être incluses dans une étude traitant de l'esthétique vocale. De même, les voix dysphoniques sévères (G3), connues pour être régulièrement jugées comme désagréables (Altenberg \& Ferrand, 2006 ; Raymond, 2010) ont été écartées.

Tab. 3. Causes et effectifs des pathologies vocales

\begin{tabular}{|l|l|}
\hline Lésions & Effectifs /31 \\
\hline Polypes & $n=12$ \\
\hline Paralysie laryngée & $n=4$ \\
\hline Granulome & $n=1$ \\
\hline
\end{tabular}




\begin{tabular}{|l|l|}
\hline Kyste & $n=1$ \\
\hline Sulcus & $n=1$ \\
\hline Nodule & $n=1$ \\
\hline Parésie + atrophie & $n=1$ \\
\hline Edème de Reinke & $n=1$ \\
\hline Fuite ovalaire & $n=1$ \\
\hline Larynx normal & $n=5$ \\
\hline
\end{tabular}

\subsection{Expérience perceptive}

16 Afin de réduire la durée de l'expérience perceptive, 34 des 62 échantillons de voix disponibles ont été sélectionnés de façon aléatoire pour servir de stimuli lors d'une expérience perceptive $e^{3}$, soit 17 voix G0, 10 voix G1 et 7 voix G2. En outre, 6 extraits de ces 34 échantillons ont été présentés par deux fois de sorte à mesurer, pour chaque juge, la cohérence de ses réponses. Les 40 extraits (d'une durée de 20 secondes en moyenne) ont ensuite été présentés à un jury naïf composé de 92 femmes âgées de 20 à 60 ans $^{4}$ (âge moyen $=35$ ans $\pm 12,6$ ) par le biais d'une interface graphique de programmation conçue $a d$ hoc et permettant la récupération automatique des réponses sous format Excel. La durée du test était d'environ 40 minutes. Après avoir renseigné un questionnaire sociologique fournissant des informations relatives à l'âge, à la profession et au niveau d'études, les participantes avaient pour tâche de juger le caractère plus ou moins séduisant des voix entendues sur une échelle à 6 points, où 1 signifie que la voix entendue n'est « absolument pas séduisante », 2 «pas du tout séduisante », 3 «pas séduisante », où 4 que la voix est «plutôt séduisante », 5 « très séduisante » et 6 " extrêmement séduisante ».

Plusieurs arguments peuvent être avancés pour justifier le choix d'une telle échelle ; mais nous ne citerons ici que les plus signifiants pour notre propos et renverrons le lecteur désireux d'en savoir plus à l'exposé approfondi de Révis (2004) : d'une part l'évaluation du caractère séduisant d'un objet complexe tel que la voix induit plusieurs degrés, les échelles bipolaires s'avérant ainsi peu pertinentes, d'autre part, nous savons que si les échelles visuelles analogiques permettent d'obtenir des différences fines, elles favorisent la sensibilité au détriment de la concordance interindividuelle. Enfin, les échelles de classe proposant une position neutre conduisent fréquemment les sujets à ne pas trancher. Les écoutes ont été réalisées individuellement à un niveau d'écoute confortable dans un environnement calme à l'aide d'un ordinateur et d'un casque audio. Afin de ne pas influencer leurs réponses, les auditrices ont réalisé le test «à l'aveugle » : aucune d'entre elles ne savait que des voix normales et pathologiques leur seraient présentées. 


\section{Résultats}

\subsection{Au plan perceptif}

Les évaluations moyennes attribuées pour chaque grade de dysphonie (Tableau 4) et pour chacune des composantes de l'échelle GRBAS étudiées (i.e. $\mathrm{R}$ et $\mathrm{B}$ ) sont présentées cidessous (Tableaux 5 et 6 ). Il convient par ailleurs de noter que l'analyse de la cohérence des réponses intra sujet ne permet pas d'observer de différence significative lors de l'évaluation des 6 échantillons vocaux répétés $(\mathrm{p}=0.8)$.

Tab. 4. Évaluation moyenne en fonction du grade (G)

\begin{tabular}{|l|l|l|}
\hline Grade & Évaluation moyenne /6 & e-t \\
\hline G0 & 3.52 & 1.11 \\
\hline G1 & 3.24 & 1.13 \\
\hline G2 & 2.76 & 1.24 \\
\hline
\end{tabular}

Tab. 5. Évaluation moyenne en fonction du degré de raucité (R)

\begin{tabular}{|l|l|l|}
\hline Grade & Évaluation moyenne /6 & e-t \\
\hline$R 0$ & 3.43 & 1.15 \\
\hline$R 1$ & 3.17 & 1.13 \\
\hline$R 2$ & 2.70 & 1.23 \\
\hline
\end{tabular}

Tab. 6. Évaluation moyenne en fonction du degré de souffle (B)

\begin{tabular}{|l|l|l|}
\hline Grade & Évaluation moyenne /6 & $\mathrm{e}-\mathrm{t}$ \\
\hline B0 & 3.44 & 1.15 \\
\hline B1 & 3.20 & 1.13 \\
\hline B2 & 2.81 & 1.23 \\
\hline
\end{tabular}

19 Le grade de dysphonie (G), la raucité (R) et le souffle (B) ont un effet significatif sur les évaluations : globalement, plus les voix sont dysphoniques, rauques et/ou soufflées, plus les appréciations des juges sont négatives $(\mathrm{p}<.05)$. Des tests multiples (ANOVA en mesures répétées, Test de Tuckey réalisé à l'aide du logiciel SAS version 9.2) ont été effectués en post-hoc, afin de mettre en évidence les degrés de grade, de raucité et de souffle ayant donné lieu aux meilleures évaluations. 
20 En ce qui concerne le grade, les appréciations attribuées aux voix G0 sont significativement meilleures que celles attribuées aux voix dysphoniques de grades 1 et 2 (respectivement $\mathrm{p}<.0001$ et $\mathrm{p}<.0001$ ). De même, la comparaison des évaluations attribuées aux voix dysphoniques en fonction du degré de sévérité de la pathologie indique que les voix G1 sont significativement jugées comme plus séduisantes que les voix G2 ( $\mathrm{p}<.0001)$.

Pour le paramètre de raucité, les résultats révèlent que les voix dysphoniques $\mathrm{R} 1$ sont perçues comme plus séduisantes que les voix R0 ( $p=.0001)$ que les voix R2 ( $p=.0026)$, les voix saines n'obtenant pas - sur la base de ce seul critère - de meilleures évaluations que les voix pathologiques $(\mathrm{p}=.6563)$.

22 Enfin, pour le souffle, les résultats montrent que les voix B0 sont mieux évaluées que les voix B1 et B2 (respectivement $p<.0001$ et $p<.0001$ ), les voix légèrement soufflées (i.e. B1) étant significativement mieux évaluées que les voix B2 ( $p=0.0016)$.

\subsection{Au plan acoustique}

23 Afin de vérifier ce qui distingue les différents groupes de locuteurs, des mesures de la fréquence fondamentale (F0) ont été prises sur le texte lu par les locuteurs sains recrutés dans le cadre de cette expérience, ainsi que sur les voyelles tenues [a] produites par les locuteurs dysphoniques, et cela à l'aide du logiciel Praat. Cette mesure avait pour objectif de vérifier si les juges étaient davantage sensibles à la hauteur de la voix (résultat fréquemment observé dans la littérature) ou si leur jugement était lié au traitement d'autres aspects de la voix. Parallèlement à cela, le Harmonics-to-Noise Ratio (HNR) a également été quantifié, dans le but d'observer si ce paramètre, qui donne une indication sur le rapport bruit/harmoniques dans une voix, était pertinent pour qualifier une voix jugée séduisante. Pour interpréter cette valeur, rappelons plus le ratio baisse, plus le signal est envahi par le bruit, la voix est alors plus rauque. Le jitter, qui est une mesure des perturbations à court terme de la fréquence fondamentale du signal sonore, a également été quantifié. Notons que, même si un grand nombre de mesures sont réalisables sur la voix, nous n'en avons retenu que trois dans le cadre de cette étude afin d'évaluer si les auditrices sont plus sensibles à la hauteur de la voix ou à son timbre.

Cette partie a pour principal objectif de vérifier - au plan acoustique - ce qui permet de distinguer les voix R1, qualifiées de séduisantes, des voix R2 qui n'ont pas été perçues ainsi. En d'autres termes, nous cherchons à savoir si cette préférence est sous tendue par certains paramètres acoustiques et, le cas échéant, lesquels. Signalons qu'une première étude de la fréquence fondamentale, du HNR et du jitter sur le texte de "La Chèvre de Monsieur Seguin » lu par l'ensemble des sujets a été préalablement réalisée, dans la mesure où chacun des locuteurs enregistrés a produit les mêmes séquences sonores. De ce fait, des différences entre les groupes testés pouvaient être attendues en parole lue. En effet, si d'ordinaire, ces mesures se pratiquent principalement sur des voyelles tenues, il s'agissait d'essayer de trouver un lien direct entre le jugement des auditrices lors de l'expérience en perception et les paramètres acoustiques des voix soumises à évaluation. Les mesures obtenues ne se sont pas révélées significatives, dans le sens où elles n'ont pas permis de distinguer les différents groupes de voix sur le plan acoustique. Bien que non significatives au plan statistique pour discriminer les groupes, les valeurs observées au niveau acoustique pour le F0 et le jitter sont présentées à titre indicatif dans le tableau 7. 
Tab. 7. Valeurs moyennes (et écart-type) pour le HNR, le jitter et le F0 en fonction du grade de dysphonie

\begin{tabular}{|l|l|l|l|}
\hline Grade de dysphonie & HNR & Jitter (e-t) & F0- centre de V (e-t) \\
\hline G0 & 12,03 & $2,54(1,15)$ & $121(27)$ \\
\hline G1 & 12,43 & $1,85(0,88)$ & $127(29)$ \\
\hline G2 & 11,98 & $2,67(2,47)$ & $140(35)$ \\
\hline
\end{tabular}

Compte tenu des difficultés rencontrées pour la mesure des dyspériodicités vocales en parole connectée, nous avons été amenés à étudier l'instabilité du signal de façon classique, c'est-à-dire sur des voyelles soutenues (Tableau 8). Bien que non écologique, cette condition présente l'avantage de faire émerger des différences entre les groupes. Par conséquent, si la suite de cette partie ne porte que sur des voyelles [a] prolongées, il est évident qu'un enjeu majeur serait de pouvoir réaliser ce type d'analyses sur des échantillons de parole continue.

Tab. 8. Valeurs moyennes (et écart-type) pour le HNR, le jitter et le F0 en fonction du degré de raucité

\begin{tabular}{|l|l|l|l|}
\hline Degré de Raucité & HNR & Jitter (e-t) & F0- centre de V (e-t) \\
\hline R1 & $22(3)$ & $0,65(0,14)$ & $123(20)$ \\
\hline R2 & $14(4)$ & $1,07(0,28)$ & $127(35)$ \\
\hline
\end{tabular}

Les mesures de la fréquence fondamentale n'ont pas révélé de différences pertinentes entre les deux groupes. En effet, le F0 est de $123 \mathrm{~Hz}$ (écart-type : $20 \mathrm{~Hz}$ ) en moyenne pour le groupe R1, alors que ce même paramètre a été quantifié à $127 \mathrm{~Hz}$ (écart-type : $35 \mathrm{~Hz}$ ) pour les locuteurs R2. Par conséquent, F0 ne semble pas prépondérant dans le choix des auditrices puisque, d'une part, ce critère ne permet pas de discriminer les deux groupes de voix et, d'autre part, parce que la hauteur de voix ne semble pas altérée outre mesure. La littérature (Vaissière, 2006, par exemple) a en effet montré que les valeurs moyennes de F0 étaient généralement comprises entre $100 \mathrm{~Hz}$ et $150 \mathrm{~Hz}$ pour les voix d'hommes. De ce fait, si la hauteur de la voix ne semble pas revêtir une dimension essentielle dans le jugement des auditrices, il semble intéressant d'observer des résultats portant plus directement sur la qualité du timbre vocal.

Si la fréquence fondamentale ne permet pas de distinguer les groupes R1 et R2, il n'en est pas de même pour le jitter. En effet, ce paramètre a été évalué à $0,65 \%$ pour le groupe de locuteurs classés R1 alors qu'il est à 1,07\% pour les sujets se trouvant dans le groupe R2, le seuil généralement admis pour distinguer une voix normale d'une voix pathologique étant à 1,04 \% dans la littérature (Baken \& Orlikoff, 2000 ; Boersma \& Weenink, 1996). En résumé, le jitter, qui délivre une indication sur le degré de perturbation de Fo, est plus élevé et se trouve sensiblement au-dessus de la limite servant généralement à parler de 
voix pathologiques pour le groupe $\mathrm{R} 2$ tandis que les sujets $\mathrm{R} 1$ attestent des valeurs se situant dans la norme.

Le troisième paramètre étudié, le HNR, montre également des différences entre les groupes R1 et R2. Ce ratio est de 22 (e.-t. : 3) pour les locuteurs légèrement dysphoniques alors qu'il est de 14 (e.-t. : 4) pour les locuteurs classés R2. De ce fait, le niveau de bruit dans la voix est plus élevé pour le second groupe par rapport au premier, laissant entendre une raucité plus importante pour les locuteurs R2. Du point de vue clinique, la raucité traduit l'impossibilité d'une partie ou de toute la muqueuse de la corde vocale, à se laisser déformer, sous la forme d'une ondulation, au passage des puffs d'air expiratoire. Pour le praticien, la raucité vocale est évocatrice d'une rigidité. Néanmoins, pour le juge naïf, nos résultats suggèrent qu'il s'agit d'un paramètre perçu comme séduisant jusqu'à un certain degré et moins esthétique au-delà de certaines valeurs. Il convient dès lors d'étudier plus avant la question du seuil de raucité afin de déterminer plus précisément le passage d'une voix sensuellement rauque à une voix pathologiquement rocailleuse. Ces résultats, obtenus sur un nombre relativement important de voix (i.e. $n=34$ ) peuvent en effet autoriser des comparaisons intéressantes avec d'autres études similaires.

\section{Conclusion}

La phonation est un phénomène complexe, dont les mécanismes biomécaniques sont encore incomplètement cernés. Une altération de la voix peut entrainer d'importantes perturbations dans la communication, mais aussi dans l'identité et la représentation sociale du patient. Le degré de sévérité de la pathologie semble également avoir un effet certain sur l'appréciation du caractère plus ou moins séduisant des individus à partir des seules caractéristiques vocales, tout du moins pour ce qui est du grade et du souffle. En revanche, pour ce qui concerne la raucité, nos résultats montrent que ce paramètre constitue, dans une certaine mesure, un facteur de séduction vocale. Bien que nous soutenions énergiquement que la raucité doit être considérée comme un symptôme sérieux de maladie, il semblerait que les voix légèrement rauques soient - en ce début de $21^{\circ}$ siècle - promues au rang de nouvelle esthétique vocale.

Les auteurs tiennent à remercier très chaleureusement toutes les personnes qui ont accepté de participer bénévolement à cette recherche en prêtant très gentiment leur voix ou en accordant de leur temps pour participer à l'expérience en perception.

\section{BIBLIOGRAPHIE}

Allaine, D. \& F., Cezilly (2010) Évolution et typologie des régimes d'appariement in Thomas, F ., Lefèvre, T., Raymond, M. (Eds.), Biologie évolutive, p. 423-452.

Altenberg, E. \& C. Ferrand (2006) Perception of individuals with voice disorders by monolingual English, bilingual Cantonese-English and bilingual Russian-English women, Journal of Speech, Language and Hearing Research, 49, p. 879-887. 
Apicella, C. L., Feinberg, D. R., Marlowe, F. W. (2007) Voice pitch predicts reproductive success in male hunter-gatherers, Biology Letters, 3, p. 682-684.

Apple, W., Streeter, L. A., Krauss, R. M. (1979) Effects of pitch and speech rate on personal attributions, Journal of Personality and Social Psychology, 37, p. 715-727.

Baken, R. J., \& R. F. Orlikoff (2000) Clinical measurement of speech and voice (2nd ed.), San Diego, CA: Singular Publishing Group.

Barkat-Defradas, M. \& F. Dufour (2007) La mimesis vocale : un phénomène dialogique ?, in Barbéris, J-M. (éd.), Cahiers de Praxématique, 49, p. 57-77.

Barkat-Defradas, M. \& M. Raymond (2011) Speech tempo: an honest signal for selecting mate for reproduction? Paper presented at the $23^{r d}$ Annual Conference of Human Behavior \& Evolution Society, June 29 - July 3, 2011, Montpellier (France).

Barberis, J.-M. \& M. Barkat-Defradas (Sous presse) La parole chansonnière, de Montmartre à Montmertre : le rôle des cabarets dans une mode langagière fin de siècle. in Siouffi, G. et al. (éds.), Modes langagières dans l'histoire. Processus mimétiques et changements linguistiques. Paris: Champion.

Bele, I. V. (2004) Reliability in Perceptual Analysis of Voice Quality, Journal of Voice, 19, 4, p. 555573.

Benjamin, G. R. (1981) Interactional uses of tone of voice in the United States and Japan. Semiotica, 35 , p. 71-92.

Berry, D. S. (1990) Vocal attractiveness and vocal babyishness: Effects on stranger, self and friend impressions, Journal of Nonverbal Behavior, 14, p. 141-153.

Boersma, P., \& D. Weenink (1996) Praat, a system for doing phonetics by computer, version 3.4. Institute of Phonetic Sciences of the University of Amsterdam, Report 132. 182 pages.

Boula De Mareuil, P., Rilliard, A., Allauzen, A. (2008) A diachronic study of prosody through French audio archives, International Conference: Speech Prosody 2008, Campinas, Brazil, May 6-9, 2008, p. 531-534.

Braun, A. (1996) Age estimation by different listener groups. The Journal of Speech Language and the Law, 3, 1, p. 65-73.

Brown, B., Strong, W., Rencher, A. (1973) Perceptions of personality from speech: Effects of manipulations of acoustical parameters, Journal of the Acoustical Society of America, 54, p. 29-35.

Bruckert, L., Lienard, J. S., Lacroix, A., Kreutzer, M., Lebourcher, G. (2006) Women use voice parameters to assess men's characteristics, Proceedings of Biological sciences, 273, 1582), The Royal Society of London, p. 83-293.

Bruckert, L., Bestelmeyer, P., Latinus, M., Rouger, J., Charest, I., Rousselet, G, Kawahara, H., Belin, P. (2010) Vocal Attractiveness Increases by Averaging, Current Biology 20, p. 116-120.

Ciceri, R. (2001) Seductive Communication: Paradoxical Exhibition, Obliquity and nonverbal Synchorization., in Anolli, L., Ciceri, R., Riva, G. (eds), New perspectives in Miscommunication,), Amsterdam: IOS Press, P. 106-118.

Collins, S. A. (2000) Men's voices and women's choices. Animal Behaviour, 60, p. 773-780.

Collins, S. A., Missing, C. (2003) Vocal and visual attractiveness are related in women. Animal Behaviour, 65, p. 997-1004.

Cornut, G. (2005) nouvelle édition 2009) La voix, Paris : Presses Universitaire de France, Collection Que Sais-je ?, 627. 
De Bodt, M. S., Van De Heyning, P. H., Wuyts, F. L., Lambrechts, L. (1996) The perceptual evaluation of voice disorders, Acta Otorhinolaryngol, 50, p. 283-91.

Ellis, D. S. (1967) Speech and social status in America, Social Forces, 45, p. 431-437.

Evans, S., Neave, N., Wakelin, D. (2006) Relationships between vocal characteristics and body size and shape in human males: An evolutionary explanation for a deep male voice, Biological Psychology, 72, p. 160-163.

Evans, S. (2008) The male voice: an evolutionary perspective, Doctoral thesis for the degree of doctor oh Philosophy (PhD), University of Northumbria.

Feinberg, D. R., Jones, B. C., Debruine, L. M., Moore, F. R., Law,Smith, M. J., Cornwell, E., Tiddeman, B. P., Boothroyd, L. G.,Perrett, D. I. (2005) The voice and face of woman: One ornament that signals quality? Evolution and Human Behavior, 26, p. 398-408.

Feinberg, D. R., Debruine, L. M., Jones, B. C., Little, A. C., (2008) Correlated preferences for men's facial and vocal masculinity, Evolution and Human Behavior, 29, p. 233-241.

Garnier M., Henrich N., Dubois D., Castellengo M., Poitevineau J., Sotiropoulos D., (2005) Étude de la qualité vocale dans le chant lyrique, SCOLIA, 20, p. 151-169.

Ghio, A., Teston, B., Viallet, F., Jankowski, L., Purson, A., Duez, D., Locco, J., Legou, T., Pinto, S., Marchal, A., Giovanni, A., Robert, D., Revis, J., Fredouille, C., Bonastre, J.F., Pouchoulin, G., Nguyen, N. (2006) Corpus de parole pathologique, état d'avancement et enjeux méthodologiques, Travaux Interdisciplinaires du Laboratoire Parole et Langage d'Aix-en-Provence (TIPA), 25, p. 109-126.

Ghio, A., Pouchoulin, G., Giovanni, A. (2007) Approches complémentaires pour l'évaluation des dysphonies : bilan méthodologique et perspectives, Travaux Interdisciplinaires du Laboratoire Parole et Langage d'Aix-en-Provence (TIPA), 26, p. 33-74.

Ghio, A. Giovanni, A. Teston, B., Revis, J., Yu, P., Ouakine, M., Robert, D., Legou, T. (2008) Bilan et perspectives de quinze ans d'évaluation vocale par méthodes instrumentales et perceptives, XXVIIèmes Journées d'Études sur la Parole, 9-13 juin 2008, Avignon.

Giovanni, A., Molines, V., Nguyen, N., Teston, B., Robert, D., Cannoni, M., Pech, A. (1992) Une méthode multiparamétrique d'évaluation vocale objective assistée par ordinateur, Annales d'otolaryngologie, 109, p. 200-206.

Gillie, C. (2009) « La Voix Unisexe », in Le Féminin, le masculin et la musique populaire d'aujourd'hui, Actes de la journée du 4 mars 2003. Document de recherche O.M.F. (Observatoire Musical Français), Université de Paris-Sorbonne, Paris, 2004, p. 2-17.

Gregory, S. (1994) Sounds of power and deference: Acoustic analysis of macro social constraints on micro interaction. Sociological Perspective, 37, p. 497-526.

Gregory, S., Webster, S., Huang, G. (1993) Voice pitch and amplitude convergence as a metric of quality in dyadic interviews. Language and Communication, 13, p. 195-217.

Goldstone R. L. (1998) Perceptual learning, Annual Review of Psychology, 49, p. 585-612.

Hammarberg B, Fritzell B, Gauffin J, Sundberg J, Wedin L. (1980) Perceptual and acoustic correlates of abnormal voice qualities. Acta Otolaryngology, 90, p. 441-51.

Harms, L. S. (1963) Listener comprehension of speakers of three status groups, Language Speech, 4, p. $109-112$.

Hart, R. (1971) Personality information contained in the vocal qualities and content aspects of speech, Unpublished master's thesis, Brigham Young University. 
Hartl, D. M., Crevier-Buchman, L., Vaissière, J., Brasnu, D. (2005) Phonetic effects of paralytic dysphonia, Ann. Otol. Rhinol. Laryngol. 114, 10, p. 792-798.

Hirano M. (1981) Clinical examination of voice, Springer Verlag: New York.

Hirano M. (1989) Objective evaluation of human voice: clinical aspects, Folia Phoniatrica, 41, p. 89144.

House, A \& H. B. Andrews (1987) The psychiatric and social characteristics of patients with functional dysphonia, Journal of Psychosomatic Research, 31, 4, p. 483-90.

Hughes, S.M., Dispenza, F., Gallup, G.G. (2004) Ratings of voice attractiveness predict sexual behavior and body configuration, Evolution and Human Behavior, 25, p. 295-304.

Hughes, S. M. \& G. G. Gallup, Jr. (2008) Why are we attracted to certain voices? Voice as an Evolved medium for the transmission of psychological and biological information, in Izdebski, K. (Ed.), Voice and emotion, San Diego (CA): Plural Publishing, Inc.

Horii, Y. \& W. J. Ryan (1981) Fundamental frequency characteristics and perceived age of adult male speakers, Folia Phoniatrica, 33, p. 227-233.

Hughes, S. M., Dispenza, F., Gallup Jr., G. G. (2004) Ratings of voice attractiveness predict sexual behavior and body configuration, Evolution and Human Behavior, 25, p. 295-304.

Karpf, A. (2008) La voix : un univers invisible Paris : Autrement Editions, 493 p.

Kempster, G. B., Bruce R. Verdolini, K., Barkmeier-Kraemer, J., Hillman, R. E. (2009) Consensus Auditory-Perceptual Evaluation of Voice: Development of a Standardized Clinical Protocol, American Journal of Speech-Language Pathology, 18, p. 124-132.

Konopczynski, G. (2005) Les enjeux de la voix, in Au commencement était la voix, Erès, 33-50. Konopczynski, G. (2007) La voix : monosupport ou multisupport ?, in Barbéris, J-M. (éd.), Cahiers de Praxématique, 49, p. 33-56.

Krauss, R.M., Freyberg, R., Morsella, E. (2002) Inferring speakers' physical attributes from their voices, Journal of Experimental Social Psychology, 38, p. 618-625.

Kreiman, J, Gerratt, Br, Kempster, Gb, Erman, A, Berke, G. S. (1993) Perceptual evaluation of voice quality: Review, tutorial, and a framework for future research, Journal of Speech, Language and Hearing Research, 36, p. 21-40.

Künzel, H. (1989) How well does average fundamental frequency correlate with speaker height and weight?, Phonetica, 46, p. 117-125.

Lass, N.J. \& M. Davis (1976). An investigation of speaker height and weight identification, Journal of the Acoustical Society of America, 60, 3, p. 700-703.

Lass, N. J., Phillip, J. K., Bruchey, C. A. (1980) The effect of filtered speech on speaker height and weight identification, Journal of Phonetics, 8, p. 91-100.

Laver, J. (1981) The Phonetic Description of Voice Quality, Cambridge, U.K.: Cambridge University Press.

Le Huche, F. \& A. Allali (1990) nouvelle édition 2001). Pathologies vocales d'origine fonctionnelle, Tome 2, Paris : Masson, Collection Phoniatrie.

Lieberman, P. (1984) The biology of and evolution of language, Cambridge, Massachusetts: Harvard University Press. 
Liu, X. \& Y. Xu (2011) What makes a female voice attractive? Proceedings of XVII ${ }^{\text {th }}$ ICPHS, Hong Kong, 17-21 August 2011, p. 1274-1277.

Louvet, R. (2010) Traitement des cancers débutants de la corde vocale : étude comparative des conséquences vocales et sociales, Mémoire de fin d'études en orthophonie, Université de Montpellier I, Faculté de Médecine.

Loredana, C., Mauro, F., Paoloni, A (2000) Subjective age estimation of telephonic voices, Speech Communication, 31, p. 107-112.

Mullenix, J. W., Johnson, K. A., Topcu-Durgun, M., Farnsworth, L. M. (1995) The perceptual representation of voice gender, JASA, 98, p. 3080-3095.

Neiman, G. S. \& J. A. Applegate (1990) Accuracy of listener judgment of perceived age relative to chronological age in adults, Folia Phoniatrica, 42, p. 327-330.

Payri, B. (2000) Perception de la voix parlée : cohérence du timbre du locuteur, Thèse de Doctorat de l’Université Paris XI.

Pemberton, C., Mccormack, P., Russell, A. (1998) Have Women's Voices Lowered Across Time? A Cross Sectional Study of Australian Women's Voices, Journal of Voice, vol. 12, 2, p. 208-213.

Pierrehumbert J. B., Bent T., Munson B., Bradlow A. R., Bailey J. M. (2004) The influence of sexual orientation on vowel production, Journal of Acoustical Society of America 116, 4, p. 1905-1908.

Privat, N. (2009) Dysphonie et image sociale, Mémoire de fin d'études en orthophonie, Université de Marseille II, Faculté de Médecine.

Puts, D. A., Gaulin, S. J. C., Verdolini K. (2006) Dominance and the evolution of sexual dimorphism in human voice pitch, Evolution and Human Behavior, vol. 27, p. 283-296.

Puts, D. A., Hodges, C. R., Cardenas, R.A, Gaulin, S.J.C. (2008) Men's voices as dominance signals: vocal fundamental and formant frequencies influence dominance attributions among men, Evolution and Human Behavior, 29, p. 233-241.

Ray, G. B. (1986) Vocally cued personality prototypes: An implicit personality theory approach, Communication Monographs, 53, p. 266-276.

Raymond, F. (2010) L'image sociale véhiculée par la dysphonie, Mémoire de fin d'études en orthophonie, Université de Marseille II, Faculté de Médecine.

Révis, J., Giovanni, A., Wuyts, F., Triglia, J. (1999) Comparison of different voice samples for perceptual analysis, Folia Phoniatr Logop, 51, p. 108-16.

Revis, J., Raymond, F., Privat, N., Giovanni, A. (2009) Dysphonie et image sociale. Communication orale présentée aux $3^{\circ}$ Journées de Phonétique Clinique, 4-5 décembre 2008, Aix en Provence.

Revis, J. (2004) L'analyse perceptive des dysphonies : approche phonétique de l'évaluation vocale, Thèse de doctorat Nouveau régime en Sciences du Langage, Université de Provence.

Ryan, W. J., Burk, K. W. (1974) Perceptual and acoustic correlates of aging in the speech of males, Journal of Communication Disorder, 7, p. 181-192.

Scott, S., Robinson, K., Wilson J.A., Mackenzie, K. (1997) Patient-reported problems associated with dysphonia, Clin Otolaryngol, 22, 1, p. 37-40.

Seidner, W., Büttner, M. (1999) Le caractère esthétique des voix chantantes rauques, Bulletin d'audiophonologie, Annales scientifiques de l'Université de Franche-Comté Médecine \& Pharmacie, 15, 1, p. 85-89. 
Smith, B., Brown, W. Strong, Rencher A. (1975) Effects of speech rate on personality perception, Language and Speech, 18, p. 145-152.

Smith J. S. (1992) Women in charge: Politeness and directives in the speech of Japanese women, Language and Society, 21, p. 59-82.

Smith, S. M., Shaffer, D. R. (1995) Speed of Speech and Persuasion: Evidence for Multiple Effects, Pers. Soc. Psychology Bulletin, 21, 10, p. 1051-1060.

Smyth, R., Jacob, G., Rogers, H. (2003) Male voices and perceived sexual orientation: An experimental and theoretical approach, Language in Society, 32, p. 329-350.

Sotiropoulos, D. (2004) Analyse acoustique et catégorisation d'un ensemble de qualités vocales pertinent pour la description de voix lyriques masculines, Mémoire de stage de DEA : Acoustique, Traitement du signal et Informatique appliqués à la Musique (ATIAM) : Université Paris 6 (Ircam), 64 p.

Steer, A. (1974) Sex differences, extraversion and neuroticism in relation to speech rate during the expression of emotion, Language and Speech, 17, 1, p. 80-86.

Teston, B. (2001) L'évaluation objective des dysfonctionnements de la voix et de la parole : les dysphonies, TIPA, 20, p. 169-232.

Teston, B. (document électronique) L'évaluation objective des dysphonies : État actuel et perspectives d'évolution, Laboratoire Parole et langage Université de Provence et CNRS [http:// hal.archives-ouvertes.fr/docs/00/17/35/53/PDF/2075.pdf] - consulté le 22/03/2012.

Vaissiere, J. (2006) La phonétique, Paris : Presses Universitaires de France, Collection Que Sais-Je ?, 637.

Van Domelen, W. A. \& B. H. Moxness (1995) Acoustic parameters in speaker height and weight identification: sex-specific behaviour, Language and Speech, 38, p. 267-287.

Vukovic J., Jones, B. C., Feinberg D. R., Debruine L., M., Smith, F. G., Welling L. L., Little, A. C. (2011) Variation in the perception of physical dominance and trustworthiness predicts individual differences in the effect of relationship context on women's preferences for masculine pitch and men's voices, British Journal of Psychology, 102, 1, p. 37-48.

White A, Deary, I. J., Wilson, J. A. (1997) Psychiatric disturbance and personality traits in dysphonic patients, European Journal of Disordered Communication, 32, 3, p. 307-14.

Yuasa, I. P. (2010) Creaky voice: a new feminine voice quality for young urban oriented upwardly mobile american women? American Speech, 85, 3, p. 315-337.

Zuta, V. (2007) Phonetic Criteria of Attractive Male Voices, in Proceedings of the 16th ICPhS, Saarbrücken, p. 1837-1840.

Zuta, V. (2009) Voice Pleasantness of Female Voices and the Assessment of Physical Characteristics, in Esposito, A. \& R. Vích (eds.), Cross-Modal Analysis of Speech, Gestures, Gaze and Facial Expressions, Berlin Heidelberg : Springer-Verlag, p. 116-125.

Zuckerman, M., Driver, R. (1989) What sounds beautiful is good : The vocal attractiveness stereotype, Journal of Nonverbal Behavior, 13, p. 67-82.

\section{NOTES}

1. «Ce qui sonne bien est beau » (notre traduction). 
2. Cet article est une version complétée d'un mémoire de fin d'études en orthophonie ( $c f$. Busseuil C. \& Chauvy, O., (2011). Esthétique des voix dysphoniques: une approche perceptuelle, Mémoire de fin d'études en vue de l'obtention du Certificat de Capacité d'Orthophonie, Université Montpellier 1, Faculté de Médecine, 92 p. (sous la direction conjointe de B. Amy de la Bretèque et $\mathrm{M}$. Barkat-Defradas, et avec la collaboration scientifique de J. Révis).

3. Les 31 autres stimuli ont été utilisés dans le cadre d'un second mémoire d'orthophonie mené à Marseille (cf. Cazals, E. \& Guerin, F. (2011). Influence de la dysphonie sur l'attractivité vocale. Mémoire de fin d'études en vue de l'obtention du Certificat de Capacité d'Orthophonie, Université de la Méditerranée, Aix-Marseille II, Faculté de Médecine, Marseille, France, 39 p. (sous la direction conjointe de J. Révis, B. Amy de la Bretèque et M. Barkat-Defradas).

4. Dans le cadre de l'évaluation du caractère plus ou moins séduisant de la voix, il nous a semblé important de faire correspondre au maximum les caractéristiques des juges à celles des locuteurs dont les voix devaient être jugées, certains paramètres tels que l'âge, pouvant intervenir au moment du jugement.

\section{RÉSUMÉS}

L'évaluation de la qualité vocale et la perception de sa dégradation à travers différents indices acoustiques sont des préoccupations majeures pour les professionnels de la voix impliqués dans les processus de rééducation vocale. Dans ce cadre, il convient d'accorder une attention toute particulière à l'ensemble des indices susceptibles de délivrer des informations pertinentes pour aider au diagnostic et évaluer les effets des rééducations vocales proposées. De ce fait, l'apport de la phonétique expérimentale à la pratique clinique est un fait avéré. Si l'étude que nous proposons s'inscrit dans l'évaluation de la qualité vocale, notre problématique est davantage axée sur la dimension esthétique de la voix dans le contexte spécifique des pathologies vocales. Notre objectif vise en effet à évaluer le caractère plus ou moins séduisant des voix dysphoniques. Les résultats d'évaluation perceptive montrent que les voix légèrement rauques (degré $1 \mathrm{de}$ raucité sur l'échelle GRBAS) sont jugées comme étant les plus séduisantes parmi un ensemble de voix normales et dysphoniques. Partant de ce constat, une étude acoustique a été menée sur de la parole lue et sur des voyelles tenues afin de quantifier les paramètres propres à chaque type de voix et déterminer les corrélats acoustiques des voix perçues comme étant les plus séduisantes.

Researchers as well as speech therapists are interested in acoustic cues, that allow evaluating voice quality and its degradation in order to obtain a diagnosis and to analyze the effects of a therapy. This way, experimental phonetics can be useful to the clinical practice.

This study, which is a crossed approach on pathological speech connecting phonetic and logopedic sciences, deals with the esthetic quality of dysphonic voices. The aim of this work is to evaluate if women can perceive men's altered voices as attractive. Results show that voices that are slightly rough (i.e. R1 on GRBAS scale) are evaluated as the most attractive among a set of dysphonic and non-dysphonic voices. An acoustic study has been carried out (1) to quantify the acoustic characteristics of each type of pathological voices and (2) to examine the acoustic correlates of voices that have been perceived as the most attractive. 
INDEX

Mots-clés : qualité vocale, dysphonie, évaluation perceptive, voix parlée, voyelles soutenues

Keywords : voice quality, dysphonia, perceptive evaluation, spoken speech, sustained vowels

\section{AUTEURS}

MELISSA BARKAT-DEFRADAS

Laboratoire Praxiling UMR 5267 CNRS, Université Montpellier III

Ecole d'orthophonie, Faculté de Médecine, Université Montpellier I

CORALIE BUSSEUIL

École d'orthophonie, Faculté de Médecine, Université Montpellier I

ORIANE CHAUVY

École d'orthophonie, Faculté de Médecine, Université Montpellier I

\section{FABRICE HIRSCH}

Laboratoire Praxiling UMR 5267 CNRS, Université Montpellier III

\section{CAMILLE FAUTH}

EA LInguistique Langue et PArole EA 1339, Université de Strasbourg

\section{JOANA RÉVIS}

École d'orthophonie, Faculté de Médecine, Université Marseille II

Laboratoire Parole et Langage, UMR 7309, CNRS, Université d'Aix-Marseille

\section{BENOÎT AMY DE LA BRETÈQUE}

École d'orthophonie, Faculté de Médecine, Université Montpellier I

CHU Gui de Chauliac, Service des pathologies de la voix, Montpellier I 